\title{
KINEMATICS AND DYNAMICS OF GALACTIC SYSTEMS AS PROBED BY PLANETARY NEBULAE SAMPLES
}

\author{
H. DEJONGHE ${ }^{1}$, P. VAUTERIN ${ }^{1,2}, \mathrm{~K}$. VAN CAELENBERG ${ }^{1,3}$, \\ S. DURAND ${ }^{4}$ AND A. MATHIEU ${ }^{1,4}$ \\ ${ }^{1}$ Sterrenkundig Observatorium, Universiteit Gent, Belgium \\ ${ }^{2}$ Koninklijke Sterrenwacht van België, Belgium \\ ${ }^{3}$ Research Assistant, FWO, Belgium \\ AND \\ ${ }^{4}$ Observatoire de Strasbourg, France
}

\section{Introduction}

At the current epoch, the dynamics of galactic systems is largely controlled by the behaviour of the stellar component. There is probably also a large amount of non-stellar matter present, which probably dominates the dynamics on large scales. Nevertheless, the (centers of) galaxies are still, for better or worse, defined as those regions were there happens to be a lot of stellar light.

In this contribution, we will concentrate on samples of Planetary Nebulae $(\mathrm{PNe})$. We will not try to distinguish populations. Our goal here is to obtain dynamical models with stellar distribution functions, and such models need kinematical data for at least several hundred stars, which is about the size of the best data sets available.

The reasons to consider PNe are well known. They are members of a stellar population that is thought to be dynamically relaxed and therefore suitable for modeling with equilibrium models. Their emission spectra make detection of individual stars possible, even at large distances, and allow the determination of a radial velocity. Last but not least, they can be found in the halos of galaxies, and thus provide important information on these fairly mysterious components. 


\section{KINEMATICS AND DYNAMICS OF GALACTIC SYSTEMS}

\section{Stellar dynamics}

The fundamental particle in stellar dynamics is the orbit. As such, it is certainly not the most convenient or fundamental representation. To do better, we note that in astronomy, we get but a snapshot of reality, in this case a position and a velocity at some time (at best!). Hence, ideally we need to characterize orbits by means of time-independent integrals of the motion. The fundamental question then is : how many stars are there with a given set of integrals of the motion? This is in essence the quest for the distribution function.

The choice of these integralss is not arbitrary, but follows from the equations of the motion. Therefore, the constants of the motion will always somehow refer to the forces that are locally at work. For example, when the motion is conservative there is a potential $V(\vec{r}, t)$, and when this potential is time-independent, we know that the classical energy $E=V(\vec{r})+\frac{1}{2} \vec{v}^{2}$ is conserved. Clearly, $E$ is not an observable since it contains the potential, but on the other hand, $E$ offers a means to constrain $V(\vec{r})$, and hence, e.g., the amount of dark matter. As far as the time-independent case goes, we will have occasion to refer here to models with the following symmetries : spherical symmetry, i.e. $V(|\vec{r}|)$, with integrals $E$ and $\vec{L}=\vec{r} \times \vec{v}$ in section 4.2 and 4.4; classical axisymmetry, i.e. $V(\varpi, z)$, where $\varpi^{2}=x^{2}+y^{2}$, and the integrals $E$ and $L_{z}$, in section 3.2; Stäckel axisymmetry with an additional third integral $I_{3}$ in section 3.3.

When the stellar system is time-dependent, the concept of constants or integrals of the motion loses much of its elegance, and one has to study the distribution function directly as it comes in phase space (see section 3.4)

Our goal therefore is (1) to obtain the distribution function $F$, which is the fundamental description of a stellar system. The more the system exhibits symmetry, the more fundamental this picture will be; (2) to determine some basic parameters, such as the mass (bearing on such issues as dark matter or black holes) and the angular momentum (which is an important parameter in a merger event); (3) to get an idea of the evolution, which we will here translate into the investigation of the (in)stability properties.

The means to obtain all this are twofold : (1) solving the collisionless Boltzmann equation for $F$ or using the fact that, where appropriate, $F$ is a function solely of the integrals of the motion. In that case, a fit of $F$ to the data reduces to a number of integral equations that connect $F$ to such observables as projected light density, projected velocities and dispersions. These may be solved by a Quadratic Programming technique (Dejonghe 1989), hereafter QP. In essence, one writes the distribution function as a linear combination of basis functions that are suitably chosen, and one de- 


\section{H. Dejonghe}

termines the coefficients by minimizing a sum of squares built with observables, and adding linear constraints that ensure that the solution remains positive in phase space. (2) solving the Jeans equations. This method has the advantage that it connects easily to a more familiar hydrodynamical formulation, and is readily applicable in some simple cases. On the other hand, one is never sure whether a solution will correspond to a distribution function that is positive. Both methods assume that the potential is known. By experimenting with several choices, one may obtain a best estimate for the potential.

\section{Dynamical models for the Galaxy}

\subsection{THE DATA}

$\mathrm{PNe}$ are, together with $\mathrm{OH} / \mathrm{IR}$ stars, the only stars for which samples exist that cover substantial parts of the Galaxy. For our purposes, we only consider the PNe for which radial velocities are known. The catalog by Acker et al. (1992) contains 621 entries that meet this criterion. It is, for a large part, a critical compilation of all known measurements in the literature (Schneider et al. 1983). Recently, a new catalog containing 856 PNe with radial velocities has been compiled from various sources (Durand, this volume and Durand et al. (1996), and references therein). Apart from the size, also the quality of the measurements and the estimates of the errors are greatly improved. Nevertheless, there are very important but unquantifiable selection biases present in these catalogs, since they originate in large part from literature searches. Add to this problem the galactic extinction in the plane (most of it is optical work), and it will be clear that these catalogs are unsuitable to yield star counts.

\subsection{TWO-INTEGRAL AXISYMMETRIC MODELS}

The first such models have been constructed by Durand et al. (1996). To overcome the non-kinematic detection biases, they substituded the PN counts with the $2.2 \mu \mathrm{m}$ COBE map. This emission is thought to be dominated by late- $\mathrm{K}$ and $\mathrm{M}$ giants, and is therefore fairly representative for the distribution of the galactic AGB stars.

The potential is a superposition of 2 Kuzmin-Kutuzov Stäckel potentials with a halo-disk structure, that produces a flat rotation curve, reasonable radial and thickness scalelenghts of the disk and an acceptable disk surface density at the solar radius (Batsleer \& Dejonghe 1994). The model uses $\mathrm{QP}$, and the necessary basis functions are such that thin two-integral disks are fairly easily produced (Batsleer \& Dejonghe 1995).

So far, two-integral models have been made only for the Acker et al. 

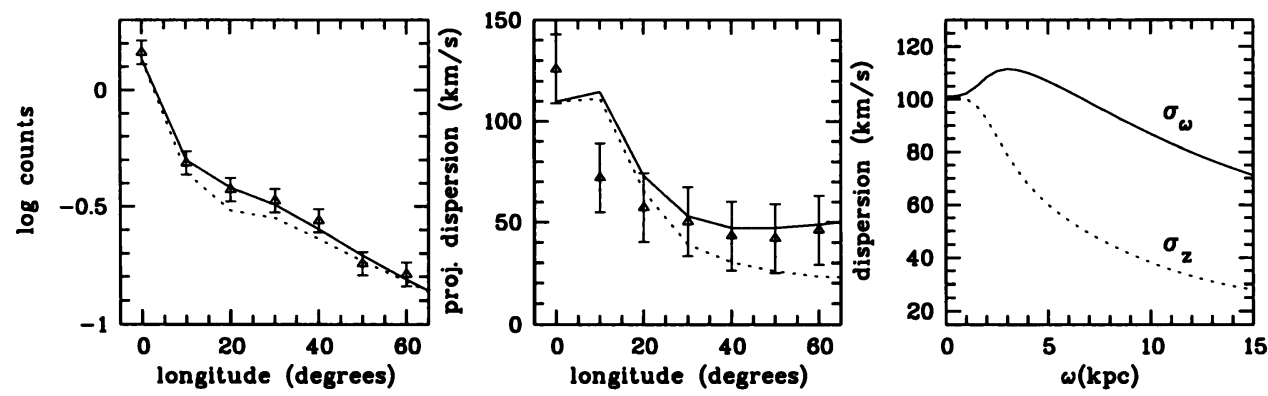

Figure 1. A comparison of a two-integral model (dotted curves) and a three-integral model (full curves), superposed on data from the Acker et al. sample. Left panel: projected star counts from the COBE map, middel panel: projected velocity dispersion, right panel: spatial deprojected velocity dispersion in $\varpi$ (dotted curve) and $z$ (full curve)

(1992) catalog (Durand et al. 1996). The fit has an overall good quality, except maybe for the projected velocity dispersion, which decreases too fast in the model (see middle panel of fig.1). This could be a natural consequence of the disk structure, which needs small intrinsic velocity dispersions in the $z$-direction $\sigma_{z}$, and the 2-integral structure, which forces intrinsic velocity dispersions in the radial direction $\sigma_{\varpi}$ to be equal to the small $\sigma_{z}$. This result they interpreted as evidence for a third integral on a global scale. As a by-product, they obtain a spatial (deprojected) density map of the stellar galaxy, which has an underlying positive distribution function, and identify and parametrize several components.

\subsection{THREE-INTEGRAL AXISYMMETRIC MODELS}

Clearly one would expect that three-integral models will not suffer from the same problems as indicated above. This is indeed the case. However, it is not completely trivial to construct such models. Not only does one need to adopt a functional form for a third integral, but the construction of fairly thin disks is in itself a challenge. Preliminary results are shown in fig.1, using three-integral disklike basis functions and the Stäckel potential mentioned above. Clearly the projected velocity dispersions are much larger now at large galactic longitude, which is also reflected in the inequality of $\sigma_{\varpi}$ and $\sigma_{z}$.

\subsection{MODELS FOR THE BAR}

We present here a model that is based on an unstable linear mode occurring in an initially axisymmetric model. The galaxy is supposed to consist of two components : (1) An unperturbed, axisymmetric and time-independent part $\left(V_{0}, f_{0}\right)$. The parameters of this models are adjusted in order to fit the 


\section{H. Dejonghe}
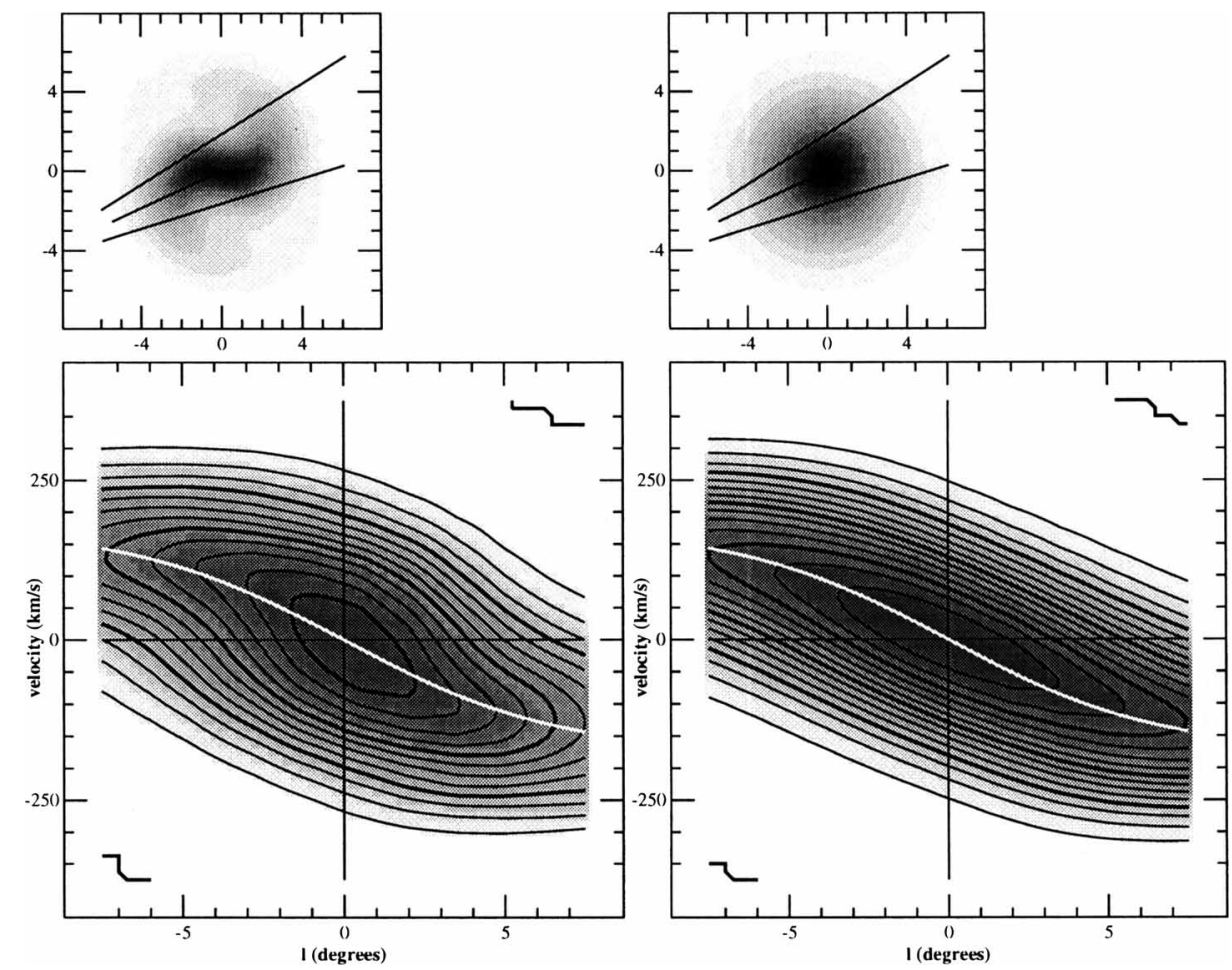

Figure 2. $\quad l-v$ diagrams of the barred model (left) and the unperturbed system (right). The axisymmetric rotation curve is indicated by the white line. The orientation of the observer of the system is shown in the top panels

central region of the Galaxy. (2) The perturbation, generally not axisymmetric and time-dependent $\left(V^{\prime}, f^{\prime}\right)$. The time- and angular dependence of these functions is supposed to be harmonic with symmetry number $m$.

The perturbation is described by the Poisson equation and the linearized Boltzmann equation. Linear modes are solutions $\left(V^{\prime}, f^{\prime}\right)$ that satisfy both equations simultaneously. The analyses show that, for each value of $m$, an infinite series of linear modes exists, with different rotation speeds and growth rates. The mode with the highest growth rate is the dominant instability, and turns out to be an $m=2$ barlike structure.

By construction, linear modes are only valid for infinitesimally small amplitudes of the perturbation. In order to construct finite amplitude perturbations, we performed a nonlinear extension. The total potential is taken from the linear mode, $V_{\text {TOT }}=V_{0}+\epsilon V^{\prime}$, but the corresponding distribution function is obtained by solving the full, nonlinear Boltzmann equation numerically. In this way, we constructed a physically meaningful perturbed model, which is self-consistent to a large degree. 


\section{KINEMATICS AND DYNAMICS OF GALACTIC SYSTEMS}

In fig. 2, we compare the stellar $l-v$ diagram of the barred model to the one of the unperturbed system. The most important difference is that the average radial velocity of the stars is higher than the rotation curve of the axisymmetric potential, which is a clear indication of triaxiality. However, if the potential is not a priori known, the barred $l-v$ diagram could well be compatible with an axisymmetric system that has a steeper rotation curve. Therefore, the $l-v$ diagram alone is usually not sufficient to prove triaxiality, and one needs to incorporate other information as well.

This barred stellar $l-v$ diagram roughly shows the same behaviour as for the gaseous component, but the features are much less pronounced. This is mainly due to the important velocity dispersion of the stars, which tends to smooth out the various properties of the different orbital families. The situation for the gas is much simpler, since, in a good approximation, the behaviour of the system at each point is completely determined by the single, closed orbit passing trough that point.

\subsection{SIMULATED SAMPLES OF PN OBSERVATIONS}

The distribution function of this model allows one to construct simulated samples of stars in the neighbourhood of the galactic centre. We now discuss the minimum sample size and the required observational accuracy in order to be able to detect triaxiality in the kinematical properties. We consider a kinematical tests for triaxiality that combines radial velocities and distance information in the following way. For $\mathrm{PNe}$, it is possible to obtain a rough distance estimate. This information can be used to divide the PNe in two classes: those that are farther away than the galactic centre (class I) and those that are not (class II). We further divide each class in two subclasses, containing stars with positive and negative galactic lengths $l$. The presence of a bar (and the fact that the sun is not oriented along one of its symmetry axes) removes the symmetry of the $l-v$ diagrams of these subclasses. We devised a test parameter to measure this asymmetry:

$$
\text { test }=\frac{1}{2}\left(\frac{\left\langle v_{I, l>0}\right\rangle}{\left\langle v_{I I, l>0}\right\rangle}+\frac{\left\langle v_{I I, l<0}\right\rangle}{\left\langle v_{I, l<0}\right\rangle}\right),
$$

where $\left\langle v_{\ldots . . .}\right\rangle$ stands for the mean radial velocity in a certain subclass. This diagnostic is independent of photometric effects, and is relatively insensitive to noise effects.

In order to determine the required minimum sample of the $\mathrm{PNe}$, we created a large number of different samples, and determined each time the value of this test parameter. In this way, we could construct its distribution, for the barred model as well as for the axisymmetric one. The results for $N=700$ and $N=1400$ sample sizes are shown in fig. 3 . For $N=700$, there 


\section{H. Dejonghe}
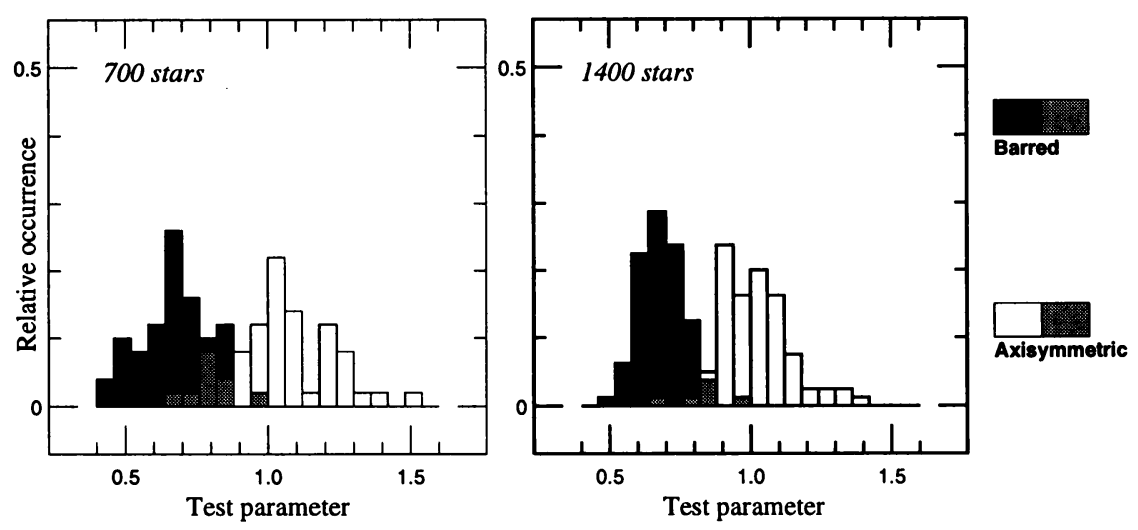

Figure 3. Monte-Carlo simulations of the test parameter (left panel: 700 stars, right panel: 1400 stars).

is still a relatively large overlap of both distributions, but the $N=1400$ samples are separated almost completely, allowing one to distinguish with a high degree of confidence between the axisymmetric or the triaxial model. We investigated various other test parameters, some of them also involving proper motion data, and they all seem to give comparable results.

These simulations show that finding kinematical evidence for triaxiality in the PN observations requires a large number of stars (1000 or more). In addition, it is not sufficient to look only at radial velocities, but one rather needs to include some other information, such as distances or proper motions.

\section{Dynamical models for extragalactic systems}

\subsection{DATA ACQUISITION}

There are a few techniques that are used to survey $\mathrm{PNe}$ in extragalactic systems. The up to now most common technique is the blinking of two images, one of them taken in a narrow band filter around [O III] ("on band"), the other in a sufficiently wide band that does not contain [O III] ("off band"). Spurious detections can be minimized by considering in addition $\mathrm{H} \alpha$ images. Subsequent multifiber spectroscopy yields the radial velocities. This method has been fully exploited by Ciardullo, Jacoby, Hui, Ford en coworkers (see e.g. Hui \& Ford 1993). Variants of this technique include the construction of colour-colour diagrams such as a (on,on-off) diagram, in combination or not with the I band, in order to select according to predefined criteria (Theuns \& Warren, preprint). A quite different approach uses a Fabry-Perot instrument (e.g. Tremblay et al. 1995). The obvious 


\section{KINEMATICS AND DYNAMICS OF GALACTIC SYSTEMS}

advantage here is that images and spectral information are obtained at the same time, while the short spectral range does not matter since one only needs to scan around [O III]. Finally, PN searches can also be performed with dedicated instruments, such as presented at this meeting by Douglas et al..

One generally assumes that a 4-meter class telescope detects $\mathrm{PNe}$ at distances up to $20 \mathrm{Mpc}$ with reasonable integration times. We will now briefly review the results obtained so far for the galaxies that have a sufficient number of $\mathrm{PNe}$ with radial velocities, such that stellar dynamical studies make sense.

\subsection{THE LEO I GROUP (NGC 3379 AND NGC 3384)}

We know 29 PNe in the E0 elliptical NGC 3379 with radial velocities (Ciardullo et al. 1993). This is not enough really to perform any very detailed dynamical study. Luckily, the detections are uniformly spread over the galaxy up to a radius of about 3.5 effective radii. There is little or no evidence for rotation. An isotropic Jaffe model or a lowered isothermal King model fit the data well. As a consequence, anisotropic two integral models with $F(E,|\vec{L}|)$, using the QP technique, do an even better job since they are more general. The models all have total masses around $1 \times 10^{11} M_{\odot}$, though the mass-to-light ratio (M/L) may easily vary in the 6 to 8 range and the velocity anisotropy is poorly constrained.

Tremblay et al. (1995) have observed its companion, NGC 3384, a SB0 galaxy. They obtained $69 \mathrm{PNe}$ with velocities. Rotation obviously is important, with $v / \sigma=1.4$ (peak rotation over central velocity dispersion). The rotation field is reasonably symmetric, but the velocity dispersion field is much less so. A fairly simple planar model based on the Jeans equations with isotropic velocity dispersions yields a mass of about $0.7 \times 10^{11} M_{\odot}$.

Perhaps the most interesting conclusion from both studies is related to the combined mass of about $2 \times 10^{11} M_{\odot}$ for both galaxies. Schneider (1985) has detected an $\mathrm{H}$ I ring surrounding both galaxies. From the circular velocity in this ring, a mass of about $6 \times 10^{11} M_{\odot}$ can be inferred. This means that may be 2 thirds of the mass of the combined system is not directly associated with any of the 2 members, but would have to reside in an (as yet unseen) surrounding halo.

\subsection{NGC 1399}

This cD galaxy was observed by Arnaboldi et al. (1994), yielding $37 \mathrm{PNe}$ with radial velocities. The purpose was to connect the kinematics as inferred from the integrated light with the kinematics of the globular cluster system. The latter have a large velocity dispersion of about $400 \mathrm{~km} / \mathrm{s}$, and indeed 


\section{H. Dejonghe}

the rising velocity dispersion profile of the PNe connect the value of 250 $\mathrm{km} / \mathrm{s}$ at the most outward points of the integrated light to the globular cluster values. The surprise was that, while the globular cluster system does not rotate, the PNe do so with peak velocity of about $290 \mathrm{~km} / \mathrm{s}$. A possible explanation is that the $\mathrm{PNe}$ are tidally distorted by the companion giant elliptical NGC 1404, while the globular clusters could belong to the original NGC 1399.

\subsection{NGC 5128}

Cen A is by far the best studied galaxy, with some 400 PN positions, magnitudes and radial velocities known (Hui et al. 1995), that cover about 4 effective radii. This is sufficiently far to have an effective handle on the halo, and sufficiently numerous to allow for fiable kinematics. The presence of minar axis rotation points towards a merger history. Models based on the isotropic Jeans equations (Hui et al. 1995) and distribution functions that account for the observed major and minor axis rotation (Mathieu et al. 1996 and Mathieu \& Dejonghe, this volume) provide fairly robust evidence that there is about twice as much mass in the halo than there is integrated visible light at 4 effective radii. The models by Mathieu et al. (1996) also allow for the first time to estimate the masses of the 2 putative merged galaxies.

\subsection{INTERGALACTIC PNE}

As already hinted at in the first paragraph, it is not at all a weird idea to search for stars in the intergalactic medium. Moreover, the outer haloes are sure to blend smoothly into the intergalactic medium, and searches for halo PNe thus may very well yield some intergalactic denizens. Two groups have so far reported positive results: Theuns \& Warren (1996) in the Fornax cluster and Arnaboldi et al. (1996) in the outer rgions of NGC 4406 (Virgo cluster).

\section{References}

Acker, A., Ochsenbein, F., Stenholm, B., Tylenda, R., Marcout, J. \& Schon, C., 1992, Strasbourg-ESO catalogue of galactic planetary nebulae, ESO

Arnaboldi, M., Freeman, K.C., Mendez, R.H., Capaccioli, M. Ciardullo, R., Ford, H., Gerhard, O., Hui, X., Jacoby, G.H., Kudritzki, R.P.\& Quinn, P.J., preprint

Arnaboldi, M., Freeman, K.C., Hui, X., Capaccioli, M. \& Ford, H., 1994, The Messenger, 76,40

Batsleer, P. \& Dejonghe, H., 1994, Astron. \& Astroph., 287, 43

Batsleer, P. \& Dejonghe, H., 1995, Astron. \& Astroph., 294, 693

Ciardullo, R., Jacoby, G.H., \& Dejonghe, H., 1993,Astroph. J., 414, 454

Dejonghe, H., 1989, Astroph. J., 343, 113 


\section{KINEMATICS AND DYNAMICS OF GALACTIC SYSTEMS}

Douglas et al., this volume

Durand, S., Dejonghe, H. \& Acker, A., 1996, Astron. \& Astroph., 000, 000

Durand S., this volume

Durand S., Acker, A., Zijlstra, A., et al., preprint

Hui, X. \& Ford, H.C., 1993, in Planetary Nebulae, IAU Symp. 155, eds R. Weinberger \& A. Acker, Kluwer, 533

Hui, X., Ford, H.C., Freeman, K.C. \& Dopita, M.A., 1995, Astroph. J., 449, 592

Mathieu, A., Dejonghe, H. \& Hui, X., 1996, Astron. \& Astroph., 309, 30

Mathieu, A. \& Dejonghe, H., this volume

Schneider, S.E., Terzian, Y., Purgathofer, A. \& Perinotto, M., 1983, Astroph. J. Suppl., 52,399

Schneider, S.E., 1985, Astroph. J., 366, 38

Theuns, T. \& Warren, S.J., 1996, Mon. Not. R. Astr. Soc., in press

Tremblay, B., Merritt, D. \& Williams, T.B., 1995, Astroph. J., 443, L5 